\title{
COLOR SEXTET QUARKS AND NEW HIGH-ENERGY INTERACTIONS *
}

\author{
Alan R. White \\ Argonne National Laboratory, Argonne, IL 60439 \\ Kyungsik Kang \\ Brown University, Providence, RI 02912
}

\begin{abstract}
We review the implications of adding a flavor doublet of color sextet quarks to $Q C D$. Theoretical attractions include - "minimal" dynamical symmetry breaking of the electroweak interaction, solution of the Strong $C P$ problem via the "heavy axion" $\eta_{6}$, and Critical Pomeron Scaling at asymptotic energies. Related experimental phenomena, which there may be evidence for, include - production of the $\eta_{6}$ at LEP, large cross-sections for $W^{+} W^{-}$and $Z^{0} Z^{0}$ pairs and very high energy jets in hadron colliders, and a hadronic threshold above which high-energy "exotic" diffractive processes appear in Cosmic Ray events.
\end{abstract}

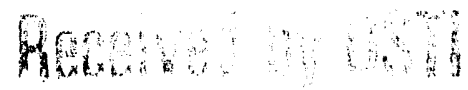

"Work supported by the U.S. Department of Energy, Division of High Energy Physics, Contract W-31-109-ENG-38.

Presented at the VIIth International Symposium on Very High Energy Cosmic Ray Interactions, Ann Arbor, Michigan, June 1992. 


\section{INTRODUCTION}

When added to $Q C D$, a flavor doublet of color sextet quarks produces a number of very attractive features, including the following.

i) A dynamical "Higgs Sector" is provided" ${ }^{1,2}$ for the electroweak sector of the Standard Model in which sextet chiral symmetry breaking by $Q C D$ is the mechanism producing electroweak symmetry breaking. That is $Q C D$ color plays the role of technicolor and the electroweak scale is actually a $Q C D$ scale. The symmetry breaking corresponds to a "minimal" Higgs sector in that " $\rho=1$ ".

ii) There is a unique resolution of the Strong $C P$ problem ${ }^{3}$ involving a very special axion - the $\eta_{6}$. This is an anomalously heavy axion that should be looked for, instead of the Standard Model Higgs particle, as a distinctive signal of sextet quark symmetry breaking.

iii) With two sextet flavors and six conventional triplet flavors we obtain a very special version of $Q C D$ with many theoretical virtues ${ }^{4}$ - including unitary Critical Pomeron scaling at high energy. In this special version of $Q C D$, "small instantons" can play an important dynamical role and may give exotic high energy diffractive interactions.

The existence of a "new" $Q C D$ sector at the electroweak scale, strongly coupled to electroweak states, would clearly have major implications for accelerator and Cosmic Ray Physics. In the latter part of this talk I shall review a number of "hints" from accelerator and Cosmic Ray experiments that this sector exists and may soon be discovered.

I would like to emphasize that from my theoretical perspective (which essentially comes from studying the consistency of $Q C D$ in the Regge limit) the sextet quark origin of a dynamical Higgs sector for the electroweak interaction is by far the most aesthetically attractive proposal for electroweak symmetry breaking.

\section{ELECTROWEAK SYMMETRY BREAKING BY COLOR SEXTET QUARKS}

In this Section we briefly review the essential features of sextet quark dynamical symmetry breaking. The original idea is due to Larciano ${ }^{1}$ and a full development of the 
effective lagrangian formalism can be found in Ref. 2 .

A massless flavor doublet $(U, D)$ of color sextet quarks with the usual quark quantum numbers (except that the role of quarks and antiquarks is interchanged) is first added to the Standard Model with no scalar Higgs sector. For the $S U(2) \otimes U(1)$ anomaly to be cancelled there must also be other fermions with electroweak quantum numbers added to the theory, but we shall not consider this issue in this paper. There is a $U(2) \otimes U(2)$ chiral flavor symmetry. We anticipate that conventional chiral dynamics will break the axial symmetries spontaneously and produce four massless pseudoscalar mesons (Goldstone bosons), which we denote as $\pi_{6}^{+}, \pi_{6}^{-}, \pi_{6}^{0}$ and $\eta_{6}$ in analogy with the usual notation for mesons composed of $u$ and $d$ color triplet quarks.

The sextet pseudoscalars have the usual Goldstone boson couplings to the sextet axial currents, which in turn couple to the electroweak gauge fields. It then follows that the $\pi_{6}^{+}, \pi_{6}^{-}$, and $\pi_{6}^{0}$ are "eaten" by the massless gauge bosons and respectively become the third components of the $W^{+}, W^{-}$and $Z^{0}$. Consequently, $Q C D$ chiral symmetry breaking generates masses for the $W^{+}, W^{-}$and $Z^{0}$ with $M_{W} \sim g F_{\pi_{6}}$ where $F_{\pi_{6}}$ is a $Q C D$ scale. An elementary picture of chiral symmetry breaking suggests a "Casimir Scaling" rule ${ }^{5}$ which can be consistent with $F_{\pi_{6}} \sim 250 \mathrm{GeV}$ !

It is important that the $\eta_{6}$ is not involved in generating mass for the electroweak gauge bosons. Instead it is an axion ${ }^{6}$, and remains massless until triplet quark masses are added via triplet/sextet four-fermion couplings. At first sight the $\eta_{6}$ also has a conventional axion mass $\sim 100 \mathrm{keV}$. However, the $\eta_{6}$ is a very special axion composed of higher color quarks. The presence of sextet quarks very significantly slows the evolution of $\alpha_{s}$ above the electroweak scale and as a result, "small instanton" interactions within $Q C D$ are a much larger effect than naively anticipated. In particular such interactions can consistently ${ }^{7,8}$ generate a much higher mass for the $\eta_{6}$.

The two photon decay rate for the $\eta_{6}$ can be calculated ${ }^{9}$ from the anomaly and for a mass of, say, $60 \mathrm{GeV}$ (we shall enlarge on the reasons for this choice below) the lifetime from this decay mode is comparable with that of the $\pi^{0}$. It is difficult to estimate the hadronic width, but if we use $\left(m_{\eta_{8}} / F_{\eta_{8}}\right)$ as an order of magnitude estimate of the amplitude involved,then we conclude that the $\eta_{6}$ should still have a significant two photon decay mode (at perhaps the $1 \%$ level) even when the mass is as high as $60 \mathrm{GeV}$. 


\section{3. $Q C D$ WITH 6 COLOR TRIPLET AND 2 COLOR SEXTET QUARKS}

As we noted in the Introduction, this theory has many interesting properties ${ }^{4}$. In particular we believe the version when all the quarks are massless is worthy of intense theoretical study - perhaps on the level which the large $N$ limit of $S U(N)$ gauge theory is studied. There are strong arguments that the massless theory has an infra-red fixed-point, implying that the gauge coupling does not grow in the infra-red region. Therefore if the conventional non-perturbative properties of confinement and chiral symmetry breaking are present, they must arise from instanton interactions - which provide the only non-perturbative ingredient of the theory.

Properties of the massless theory should clearly underlie much of the physics that sets in above the electroweak scale, as the electroweak interaction couples in to $Q C D$ via the sextet quark sector. We have already noted the importance of short-distance instanton interactions in generating the $\eta_{6}$ mass. These interactions also play an important role in the development of the high-energy forward scattering behavior of the theory. Indeed, as we argue at length elsewhere ${ }^{4}$, the infra-red fixed-point of the theory is directly reflected in Critical Pomeron scaling behavior in the asymptotic Regge limit. At the qualitative level, this suggests that instanton interactions can be thought of as directly involved in producing high-energy diffractive interactions at super-high energies.

More specifically, perhaps, an understanding of the Super-Critical Pomeron phase suggests that we can visualize the high-energy expansion of a hadron as due to a thickening "surface" full of gauge fields rich in topological structure (that is there is an effective winding number vacuum condensate in this region). Another hadron scattering peripherally through the surface region feels this structure and a variety of instanton interactions within the scattering hadron are stimulated. This would include the stimulated production of sextet quark states. As we shall return to below, this implies that SSC physics may actually be dominated by the diffractive, and multiple Pomeron production, of electroweak states - that is multiple $W^{\prime}$ s, $Z^{0}$ 's and $\eta_{6}$ 's. 


\section{POSSIBILITIES FOR DETECTION OF THE SEXTET QUARK SECTOR}

In this and the next Section we discuss possible signatures of the sextet quark sector that may, perhaps, have already been seen or may be seen in the not too distant future. We begin with accelerator phenomena in this Section and then move on to Cosmic Ray events. Since most effects we consider are non-perturbative in origin, quantitative predictions are clearly difficult.

\subsection{Perturbative $Q C D$ Production}

This can be discussed quantitatively, but unfortunately is small. The common expectation is that the constituent sextet quark mass is of $\mathrm{O}(300) \mathrm{GeV}$. This is the mass that has to be used in perturbative production calculations, via gluon fusion in particular. Apart from color factors, this calculation is the same as the production of a heavy triplet quark. Not surprisingly the total cross section is less than $1 \mathrm{~Pb}$. and so may well be too small to be seen at CDF. (Although one or two of the highest energy CDF "Zoo" events may, perhaps, be candidates!) It could be more interesting to look for higher-order gluon and quark composite operators that the sextet quark condensate will induce ${ }^{10}$. Evidence for these operators can, perhaps, already be seen in the very high-energy tail of the inclusive jet cross-section. The CDF data ${ }^{11}$ is consistent with conventional QCD but can also accommodate contributions from such operators of just the order of magnitude ${ }^{10}$ given by sextet quarks.

\subsection{Non-Perturbative Production of Electroweak States}

There will be non-perturbative production of $W^{+} W^{-}, Z^{0} Z^{0}, Z^{0} \gamma, \ldots$ states in $p p$ collisions which at high-energy will be dominantly given by (multi-) Pomeron exchange ${ }^{12}$. It is interesting that UA1 actually accumulated four $W+2$ jet events ${ }^{13}$ which are kinematically consistent with $W^{+} W^{-}$pair production and are quite distinct from the background events. These events suggest a $W^{+} W^{-}$production cross-section more than two orders of magnitude greater than that predicted by the Standard Model - with a conventional Higgs sector. CDF also has candidate $W^{+} W^{-}$and $Z^{0} Z^{0}$ events but not enough to indicate a cross-section significantly above the Standard Model prediction. Unfortunately the CDF detector does 
not cover the diffractive region where we might expect the non-perturbative production to be largest (and more easily distinguishable from background jet production).

As we mentioned above we can surely expect the diffractive production of electroweak states to be a major phenomenon at the SSC. Indeed it seems to me that it could very well become the dominant focus of SSC physics. In which case the full rapidity coverage given by a detector such as the FAD proposal ${ }^{14}$, would be an essential requirement.

\section{$4.3 \eta_{6}$ production}

In principle, the $\eta_{6}$ could be a "normal" axion, but this is ruled out by comprehensive axion searches. Fortunately, as we have already indicated, small instanton interactions can be expected to produce an anomalously high mass. As we now discuss there are experimental hints that this may be as high as $60 \mathrm{GeV}$.

The L3 experiment at LEP has now seen ${ }^{15}$ four events of the form $Z^{0} \rightarrow l^{+} l^{-}+\gamma \gamma$ where the mass of the $\gamma \gamma$ pair is close to $60 \mathrm{GeV}$. If these events indicate a new massive particle then it has a significant width, which probably has to be hadronic in origin, and a major two photon decay mode. If there is a strongly interacting massive particle radiated by the $Z^{0}$ then it is clearly very tempting to identify it with the $\eta_{6}$. At first sight, however, there is a problem ${ }^{3}$ in that rates calculated from the sextet quark anomaly ${ }^{16}$ appear to be too low to be consistent with the L3 events. However, when the axion and Strong CP properties of the $\eta_{6}$ are studied in detail ${ }^{3}$ we find that sextet quark $Q C D$ interactions need not be $C P$ conserving. As a result there are $C P$ non-conserving Goldstone boson amplitudes which can produce the L3 events consistently. The implications of identifying the L3 events with the $\eta_{6}$ will be discussed at length in Ref. 3 .

If the $\eta_{6}$ has a mass of $\mathrm{O}(60) \mathrm{GeV}$ and our diffractive enhancement picture is correct, we would expect a significant cross-section for diffractive $\eta_{6}$ production at both the CERN and Fermilab colliders. This could perhaps be correlated with the observation ${ }^{17}$ of a large real part of the forward scattering amplitude by the UA4 experiment at CERN. If the scattering is at energies well above all thresholds, the real part is related to the total cross-section by a derivative dispersion relation. Since the total cross-section is rising only relatively slowly in the same energy range, the UA4 result is inconsistent with this relation. The only viable explanation $^{18}$ is that there is a major new threshold in the strong interaction. This threshold has to be localized at roughly $\sqrt{s} 500 \mathrm{GeV}$ and could indeed be diffractive production of the 
$\eta_{6}$. We shall find further support for this suggestion in the Cosmic Ray results discussed in the next Section.

\section{HIGH-ENERGY SEXTET QUARK INTERACTIONS AND COSMIC RAY PHYSICS}

The argument that there is a major change in the nature of the strong interaction at very high Cosmic Ray energies has been made consistently over the last ten years, particularly by the Chacaltaya emulsion-chamber group ${ }^{19}$. The proportion of "exotic" events apparently rises with energy and may be as high as $50 \%$ for $\sqrt{s} \gtrsim 10 \mathrm{TeV}$. That the threshold for the lowest energy exotic events is around $\sqrt{s} \sim 500 \mathrm{GeV}$ was a major part of the argument ${ }^{20}$ for raising the CERN collider energy to search for exotic events. The "excess muon" point source events $^{21}$ also suggest a major change in the $\gamma \mathrm{p}$ interaction for $\sqrt{s} \gtrsim 1 \mathrm{TeV}$. Qualitatively these changes are consistent with what we expect from a new (very) strongly-interacting sector of $Q C D$. Also it is generally emphasized by the Cosmic Ray experimentalists that the exotic events are almost invariably diffractive in nature. This certainly fits with our expectation that diffraction will play a major role in the production of sextet quark states. It is hard to be quantitative but we can make the following observations as directly relevant to our previous discussion.

The lowest energy exotics, the "Geminions" and "mini-Centauros", have

i) a threshold of $\sqrt{s} \sim 500 \mathrm{GeV}$, ii) a common "fireball" mass (originally set at 30 $\mathrm{GeV}$ but now apparently higher for the more distinctly exotic events), iii) a cross-section which is several per cent of the total cross-section, iv) a diffractive rapidity distribution.

It is clearly tempting to identify these exotics with diffractive production of the $\eta_{6}$. We could then match the mini-Centauros with the hadronic decays of the $\eta_{6}$ and the Geminions with two-body electroweak decays (including $2 \gamma$ decays) ${ }^{3}$. These events are also characterized by large transverse momentum, as might be expected from diffractive sextet quark interactions involving small instantons. The anomalously low $\gamma$ - hadron ratio of miniCentauros could be due to the changing nature of the $\gamma p$ interaction - which we shall discuss further below.

If we consider the next class of exotic events to appear, the Chiron and Centauros, they share the following properties 
i) a threshold of $\sqrt{s} \sim 2 \mathrm{TeV}$, ii) a common fireball mass of $200-300 \mathrm{GeV}$, iii) a significant part of the cross-section, $\mathrm{iV}$ ) a diffractive rapidity distribution.

An obvious question is whether these exotics could be associated with sextet quark states. We expect an array of sextet "baryons" (involving one sextet quark and two triplet antiquarks) and vector "mesons" in the $300-1,000 \mathrm{GeV}$ mass range. It is possible that the hadronic decays of these states are dramatic (involving small instantons) and are what is seen in the higher energy exotic events.

If sextet quark interactions can account for all exotic high-energy interactions, then a significant change in the $\gamma \mathrm{p}$ interaction must be part of the picture. This is required to explain all of the anomalous shower development that is seen and the consequent identification of anomalous $\gamma$ - hadron ratios in exotic events. The photon does couple in directly to the sextet quark sector and we anticipate a very strong coupling of the Pomeron to sextet states. This will produce a much larger $\gamma p$ hadronic cross-section. Whether this will be sufficient to explain the anomalous characteristics of the exotic events - as well as the excess muon point source events ${ }^{21}$ - is, not surprisingly, a hard question to answer.

\section{REFERENCES}

1. W. J. Marciano, Phys. Rev. D21, 2425 (1980).

2. E. Braaten, A. R. White and C. R. Willcox, Int. J. Mod. Phys. A1, 693 (1986).

3. K. Kang, I. Knowles, and A. R. White, ANL/Brown preprint to appear.

4. A. R. White, Int. J. Mod. Phys. A11, 1859 (1991) and "Analytic Multi-Regge Theory and the Pomeron in QCD : II. Gauge Theory Analysis" ANL preprint - to appear, see also Nucl. Phys. B (Proc. Suppl.) 12, 190, (1990) and 25B, 167 (1992).

5. W. J. Marciano in [1], see also J. Kogut et al., Phys. Rev. Lett. 48, 1140 (1982).

6. R. D. Peccei and H. R. Quinn, Phys. Rev. Lett. 38, 1440 (1977); S. Weinberg, Phys. Rev. Lett. 40, 223 (1978); F. Wilczek, Phys. Rev. Lett. 40, 279 (1978).

7. B. Holdom and M. E. Peskin, Nucl. Phys. B208, 397 (1982). 
8. A. R. White, Mod. Phys. Letts. A2, 945 (1987)

9. T. E. Clark, C. N. Leung, S. T. Love and J. L. Rosner,Phys. Lett. B177B, 413 (1986).

10. E. H. Simmons, Phys. Lett. B259, 125 (1991).

11. CDF Collaboration, F. Abe et al., Phys. Rev. Lett. 68, 1104 (1992).

12. A. R. White, Mod. Phys. Lett. A2, 397 (1987).

13. A. Bohrer - UA1 Collaboration, Proceedings of the 8th Topical Workshop On ProtonAntiproton Collider Physics, Italy (1989) - published by World Scientific.

14. J. D. Bjorken - these proceedings.

15. O. Adriani et al.- the L3 Collaboration, CERN preprint, CERN-PPE/92-152.

16. T. Hatsuda and M. Umezawa, Phys. Lett. B254, 493 (1991).

17. UA4 Colleboration, D. Bernard et al., Phys. Lett. B198, 583 (1987).

18. K. Kang and A. R. White, Phys. Rev. D42, 835 (1990), Phys. Lett. B266, 147 (1991).

19. S. Hasegawa - these proceedings.

20. J. G. Rushbrooke, Proceedings of the XXIst International Conference on High Energy Physics, Paris (1982).

21. G. B.Yodh - these proceedings. 

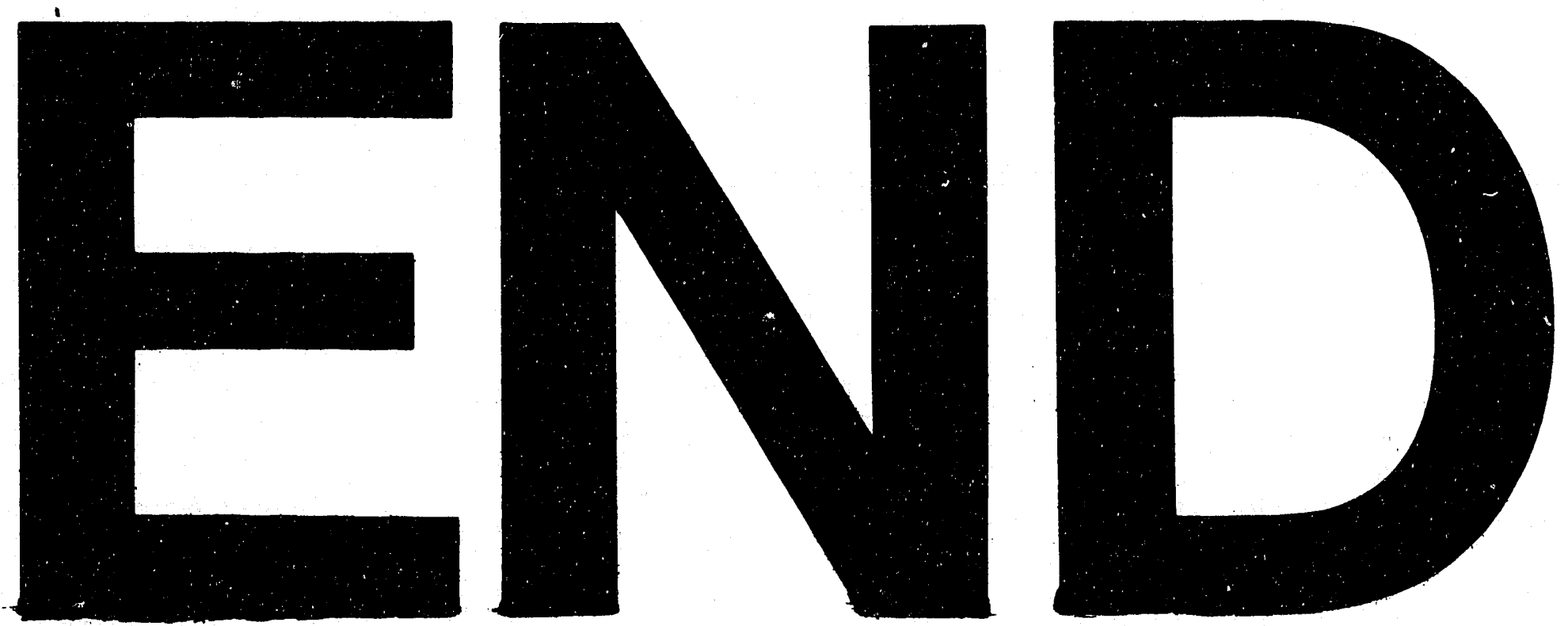

2 - Mt
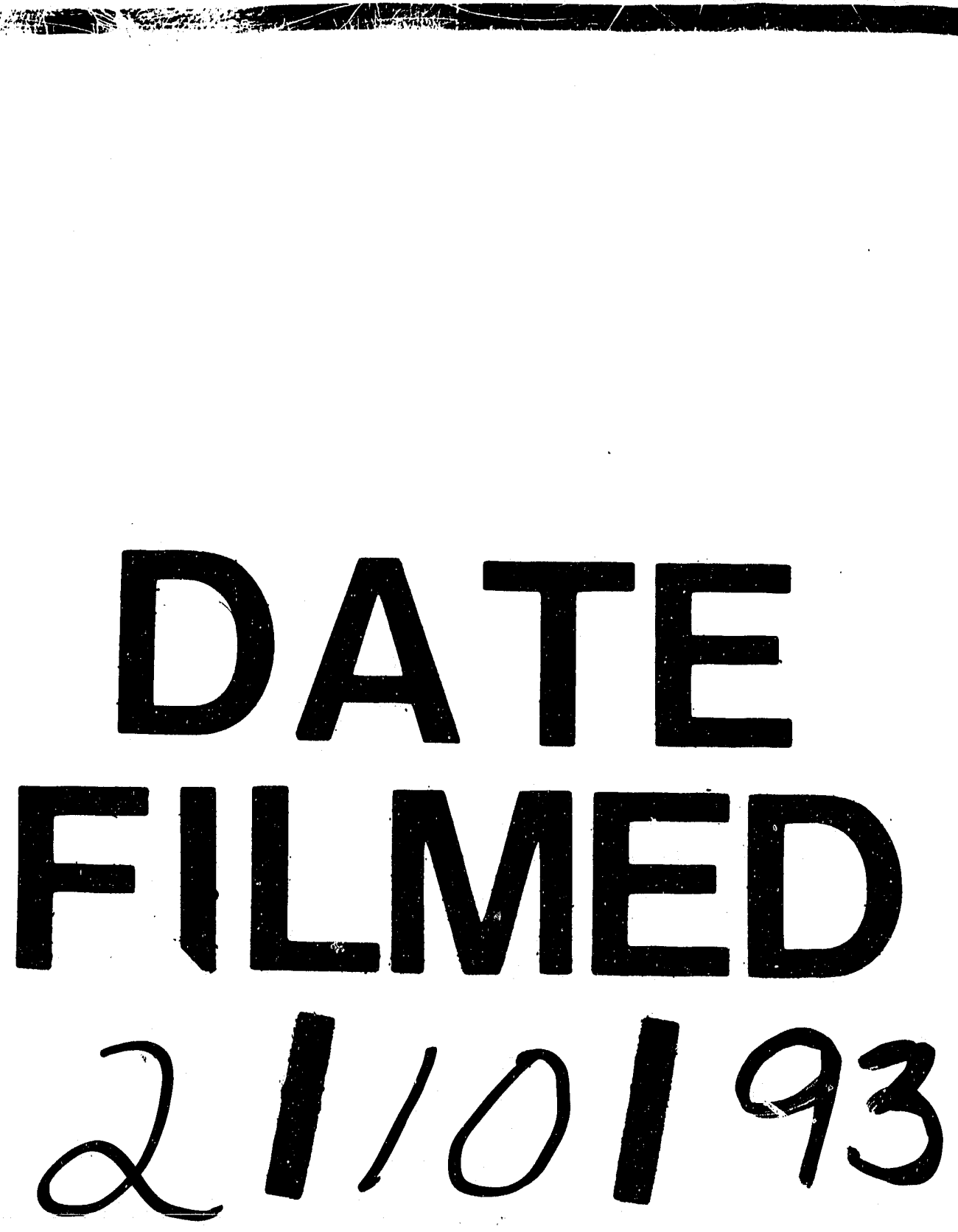
